\title{
Role of biomarkers in the diagnosis and prognosis of patients with cutaneous lupus erythematosus
}

\author{
Jane L. Zhu^, Samantha M. Black^, Benjamin F. Chong^ \\ Department of Dermatology, University of Texas at Southwestern Medical Center, Dallas, TX, USA \\ Contributions: (I) Conception and design: All authors; (II) Administrative support: BF Chong; (III) Provision of study materials or patients: None; \\ (IV) Collection and assembly of data: JL Zhu, SM Black; (V) Data analysis and interpretation: All authors; (VI) Manuscript writing: All authors; (VII) \\ Final approval of manuscript: All authors. \\ Correspondence to: Benjamin F. Chong, MD, MSCS. Associate Professor, Department of Dermatology, UT Southwestern Medical Center, 5323 Harry \\ Hines Blvd. Dallas, TX 75390-9069, USA. Email: Ben.Chong@utsouthwestern.edu.
}

\begin{abstract}
Cutaneous lupus erythematosus (CLE) is a connective tissue disease with varying presentations, and clinical sequelae including itching, dyspigmentation, and scarring. CLE can occur as its own entity or in conjunction with systemic disease, known as systemic lupus erythematosus (SLE). Because CLE is clinically diverse, identification of a biomarker may help not only facilitate early diagnosis and management but also identify individuals at risk for poor prognosis and development of SLE. While potential biomarkers in SLE have been extensively studied, few biomarkers for CLE have been identified and incorporated into clinical practice. Anti-SS-A antibody is a commonly used biomarker for diagnosis of subacute CLE patients. Type I interferon-related proteins such as MxA and guanylate binding protein-1 (GBP-1) and chemokines such as CXCR3, CXCL9, and CXCL10 have been identified as biomarkers that may support diagnosis and track disease activity. First-line oral treatment for CLE currently consists of anti-malarials such as hydroxychloroquine (HCQ), chloroquine (CQ), and quinacrine (QC). Studies have found that an increased myeloid dendritic cell population with higher TNF- $\alpha$ expression may be predictive of poor treatment response to HCQ in CLE patients. Autoantibodies against nuclear antigens (e.g., anti-double-stranded DNA and anti-Smith antibodies) and elevated erythrocyte sedimentation rate have been more commonly found in CLE patients progressing to SLE than those who have not. This review aims to summarize previous and emerging biomarkers for CLE patients.
\end{abstract}

Keywords: Biomarkers; cutaneous lupus erythematosus (CLE); systemic lupus erythematosus

Submitted Jul 11, 2020. Accepted for publication Feb 03, 2021.

doi: $10.21037 / \mathrm{atm}-20-5232$

View this article at: http://dx.doi.org/10.21037/atm-20-5232

\section{Introduction}

A biomarker is a biological entity that contributes to disease pathogenesis and reflects disease activity. Nonetheless, there are many biomarkers that can be difficult to measure and thus challenging to incorporate into clinical practice. As such, in order for a biomarker to have clinical utility, it must not only be able to accurately and sensitively respond to changes in disease activity but also be simple enough to incorporate to routine clinical practice.

Cutaneous lupus erythematosus (CLE) is an autoimmune condition with a wide range of clinical presentations. While some patients have skin-limited disease, others develop systemic symptoms and subsequently progress to systemic lupus erythematosus (SLE). CLE is clinically divided into

^ ORCID: Jane L. Zhu, 0000-0001-9823-964X; Samantha M. Black, 0000-0001-6969-6198; Benjamin F. Chong, 0000-0002-4092-7658. 
three main subtypes-acute, subacute, and chronic CLE (CCLE). Chronic cutaneous lupus can be further subdivided into discoid lupus erythematosus (DLE), lupus panniculitis, and lupus erythematosus tumidus (LET). Because CLE is a heterogenous and complex disease, clinical diagnosis and management of CLE remain a challenge. Identification of a biomarker may help not only facilitate early diagnosis but also identify individuals at risk for poor prognosis and/ or development of SLE. Given the heterogenous nature of CLE, it is unlikely that a single biomarker may be used universally for diagnosis and management. As such, there may be several relevant biomarkers. For example, some may be potentially used to facilitate accurate and early diagnosis of CLE while others may help identify individuals at risk for severe disease and poor prognosis. Finally, other biomarkers may have utility in evaluating treatment efficacy.

The utility of biomarkers in SLE has been extensively studied, as several biomarker candidates have been identified. Autoantibodies including antinuclear autoantibodies (ANA), anti-Smith (Sm) antibodies, and anti-double stranded DNA (dsDNA) antibodies have traditionally been used to diagnose $\operatorname{SLE}(1,2)$. More recently, studies have identified that SLE patients have abnormal levels of erythrocyte-bound complement activation product $\mathrm{C} 4 \mathrm{~d}$ (E-C4d) and complement receptor 1 (E-CR-1) compared to healthy patients (3). As such, E-C4d and E-CR-1 may be potential diagnostic biomarkers for SLE. Other biomarkers such as mannose binding lectin, IL-6, IL-10, and interferon-inducible chemokines (i.e., CXCL10, CCL2, and CCL19) have been found to correlate with SLE disease activity (4-6).

Despite the numerous advances that have been made in understanding CLE pathogenesis, few biomarkers for CLE have been validated and widely incorporated into clinical practice. In this review, we aim to summarize previous and more recent developments of candidate biomarkers for CLE (Table 1).

\section{Biomarkers associated with CLE and its subtypes}

Biomarkers including type I interferon-related proteins, annexin-1, and IL-18 help distinguish CLE from normal skin and other mimics of the disease. Recent studies have demonstrated that type I interferon plays an important role in driving CLE disease pathology. An immunohistochemistry study found that CLE lesions were characterized by strong expression of $\mathrm{MxA}$, a protein specifically induced by type I interferons $(9,28,29)$, and CXCR3, whose chemokine ligands are type I interferoninducible $(9,30,31)$, compared to healthy skin. Furthermore, large numbers of infiltrating CXCR3 positive lymphocytes were detected in lesional skin suggesting a Th1-based cellular immune response. CXCL10, one of the chemokine ligands for CXCR3, was found in both DLE and subacute cutaneous lupus erythematosus (SCLE) lesions and was predominantly expressed between inflammatory cells and basal layer keratinocytes and around hair follicles (9). Another study analyzed the mRNA expression of type 1 interferon-related genes from microarray data of CLE lesions ( $\mathrm{n}=90)$ and healthy controls. CLE lesional skin was found to have significantly elevated expression of two type I interferon-related genes-IFN $\alpha 10$ and IFNא compared to healthy controls regardless of CLE subtype (8). Guanylate binding protein-1 (GBP-1) is one of the most abundantly induced proteins by type I interferons and endothelial cells are known to express GBP-1 when exposed to IFN- $\alpha$ and $\gamma$, IL- $1 \alpha$ and $1 \beta$, and TNF- $\alpha$ (32-34). GBP-1 expression was determined to be upregulated in lesional skin of all CLE subtypes but not in atopic dermatitis or healthy controls (11). Finally, CLE skin lesions can histologically appear similar to dermatomyositis skin lesions, making it difficult to distinguish the two. It has recently been reported that IL-18 is uniquely elevated in dermatomyositis lesions. This cytokine in combination with $L C E 2 D, L C E 1 B$, KRT80, and TPM4 expression successfully distinguished dermatomyositis from CLE lesions (22). While these results are promising, additional studies are warranted to investigate the negative predictive value of this gene signature for CLE.

Different autoantibodies and protein biomarkers are associated with specific subtypes of CLE. SCLE is a subtype of CLE characterized by non-scarring photosensitive lesions that are most often found in upper trunk and arms. Anti-SS-A antibodies are present in approximately $63 \%$ of SCLE patients and is often used as a distinguishing feature for this subtype (26). Other CLE subtypes, particularly DLE, have not been as strongly associated with specific biomarkers as SCLE. Antibodies against annexin, an anti-inflammatory molecule that is externalized during apoptosis, have been found to be in significantly higher concentrations in the sera of 78 CLE patients $v s .51$ healthy controls. Specifically, 32\% of patients with DLE were positive for anti-annexin 1 antibodies compared with $9.7 \%$ of patients with SCLE. However, antibody levels did not correlate with disease activity (35). We previously employed 
Table 1 Candidate biomarkers for the diagnosis, management, assessment of disease activity, and prognosis of CLE

\begin{tabular}{|c|c|}
\hline Biomarker of Interest & Supportive findings \\
\hline Type I Interferon-related genes & $\begin{array}{l}\text { RNA higher in SCLE and DLE peripheral blood (7), expression correlates with disease } \\
\text { activity in SCLE and DLE patients (7) }\end{array}$ \\
\hline IFN- $\alpha 10$, IFN-א & mRNA higher in lesional CLE skin (8) \\
\hline GBP-1 & Increased protein expression in lesional skin of all CLE subtypes (11) \\
\hline CXCR3 & Increased CXCR3-expressing lymphocytes in CLE lesional skin (9) \\
\hline CXCL10 & $\begin{array}{l}\text { Increased protein expression in CLE lesional skin (9), protein expression in CLE lesional skin } \\
\text { decreases with disease activity (12) }\end{array}$ \\
\hline BAFF & mRNA higher in DLE skin (15), mRNA higher in DLE patients with concomitant SLE (15) \\
\hline VEGF, CD34 & Decreased expression after treatment with chloroquine in CLE (16) \\
\hline ESR & $\begin{array}{l}\text { Associated with concomitant diagnosis of SCLE and SLE (17), associated with concomitant } \\
\text { diagnosis of CCLE and SLE (17-19), associated with progression of DLE to SLE (20) }\end{array}$ \\
\hline Low complement, Rheumatoid factor & $\begin{array}{l}\text { Associated with concomitant diagnosis of SCLE and SLE (17), associated with concomitant } \\
\text { diagnosis of CCLE and SLE (17-19) }\end{array}$ \\
\hline Anti-dsDNA antibody & $\begin{array}{l}\text { Associated with concomitant diagnosis of SCLE and SLE (17), associated with concomitant } \\
\text { diagnosis of CCLE and SLE (17-19,23), associated with progression of CLE to SLE (24) }\end{array}$ \\
\hline Anti-annexin antibody & Increased in sera of CLE patients (26) \\
\hline Anti-SS-A antibody & $\begin{array}{l}\text { Increased in sera of SCLE patients (22), associated with concomitant diagnosis of SCLE } \\
\text { and SLE (17), associated with concomitant diagnosis of CCLE and SLE }(17,18)\end{array}$ \\
\hline Anti-U1 RNP antibody, Anti-smith antibody & $\begin{array}{l}\text { Associated with concomitant diagnosis of SCLE and SLE (17), associated with concomitant } \\
\text { diagnosis of CCLE and SLE (17-19), associated with progression of CLE to SLE (27) }\end{array}$ \\
\hline
\end{tabular}

ANA, anti-nuclear antibody; BAFF, B-cell activating factor; $\mathrm{CH} 50, \mathrm{CH} 50$, total hemolytic complement ; CLE, cutaneous lupus erythematosus; DLE, discoid lupus erythematosus; DM, dermatomyositis; dsDNA, double-stranded DNA; ESR, erythrocyte sedimentation rate; GBP-1, guanylate binding protein-1; HERC-5, hect domain and RCC1-like domain 5; IFN, interferon; ISG-15, interferon-induced protein 15; RNP, ribonucleoprotein, SCLE, subacute cutaneous lupus erythematosus; SLE, systemic lupus erythematosus; VEGF, vascular endothelial growth factor. 
autoantigen arrays to examine autoantibody profiles of healthy controls, DLE patients without SLE (DLE+SLE-) patients, SLE patients without DLE (DLE-SLE+ patients), and DLE patients with SLE (DLE+SLE+ patients) (36). Although no autoantibodies were distinctively elevated in DLE patients, increased IgG:IgM ratios of autoantibodies against nuclear antigens progressively increased from healthy controls, DLE+SLE- patients, DLE+SLE+ patients, and finally DLE-SLE+ patients. These autoantibodies seemed to correlate with disease severity in these groups of lupus patients. BAFF (B-cell activating factor) aids in $B$ cell survival and homeostasis and has been shown to be elevated in SLE patients, driving abnormal B cell development (15,37-40). Its mRNA levels have been found to be significantly higher in DLE lesional skin compared to psoriasis and healthy controls suggesting that BAFF may be a potential biomarker that can be used to distinguish DLE from other diseases (13). TNF- $\alpha$ has previously been reported to be substantially increased in the sera of SLE and CLE patients. In one study, TNF- $\alpha$ was found to be significantly elevated in PBMCs from DLE patients compared to healthy controls, but not in PBMCs from SCLE patients or LET patients (7).

\section{Biomarkers associated with CLE disease activity and treatment response}

Biomarkers such as type I interferon-inducible proteins have been shown to reflect disease activity in CLE. A previous study found that patients with SCLE and DLE had increased type I interferon-regulated gene expression compared to healthy controls regardless of concomitant SLE. Interestingly, LET patients did not have an elevated interferon signature compared to controls. Patients were assigned an interferon score based on blood expression level of five type I interferon-regulated genes previously shown to correlate with disease activity in SLE patients. This study determined that interferon scores correlated with cutaneous disease activity, suggesting its potential role as a biomarker for CLE activity (10). In a clinical trial investigating the efficacy of BIIB059, a monoclonal antibody targeting blood DC antigen 2 (BDCA2) in SLE patients, MxA skin expression was used as a marker of disease response. Skin biopsies from active lesions from SLE patients were evaluated at baseline and week 4 for IFN-regulated proteins MxA and IFITM3 using immunohistochemistry. Four weeks after receiving BIIB059 administration, 6 of 7 patients had a marked reduction in MxA percentage area immunoreactivity (12). Another clinical trial investigated the utility of anti-IFN- $\alpha$ monoclonal antibody in treating SLE patients. A panel comprised of $21 \mathrm{IFN}-\alpha / \beta$-inducible genes was used as a pharmacodynamic biomarker in this study. Skin lesions from 16 SLE patients were collected prior to treatment and compared to biopsies collected 14 days post-treatment. Baseline lesions were found to have overexpression of IFN- $\alpha / \beta$-inducible genes. Immunostaining for $3 \mathrm{IFN} \alpha /$ $\beta$-inducible proteins, hect domain and RCC1-like domain 5 (HERC-5), interferon-induced protein 15 (ISG-15), and CXCL10 was performed and revealed decreased expression of proteins in lesional skin post-treatment compared to pre-treatment (41). In DLE patients, a positive correlation has been observed between disease activity as measured by Cutaneous Lupus Erythematosus Disease Area and Severity Index (CLASI), and peripheral blood mononuclear cell TNF- $\alpha$ protein levels and sera anti-ribonucleoprotein (RNP) IgG antibody levels $(7,16)$.

CLE skin biomarkers have altered levels correlating with anti-malarial response and non-response. Firstline oral treatment for CLE consists of anti-malarials, including hydroxychloroquine (HCQ), chloroquine (CQ), and quinacrine (QC). A study of 10 patients with DLE had lesional skin biopsies at baseline and after 3 months of treatment with oral CQ. Skin expression of vascular endothelial growth factor (VEGF), a cytokine involved in angiogenesis, and CD34, an antigen expressed on endothelial cells of blood vessels, were measured by skin biopsy immunohistochemistry. After 3 months of CQ treatment, DLE skin showed reduced erythema, number of surrounding telangiectasias, and lesion size while scarring and skin atrophy persisted. Compared to baseline skin biopsies, VEGF expression was significantly reduced posttreatment and CD34+ blood vessels were smaller and less prevalent, suggesting that VEGF and CD34 may potentially be markers of successful CQ treatment in patients with DLE (42).

Approximately $50 \%$ of CLE patients are not responsive to HCQ monotherapy $(14,43,44)$. Those who do not respond to HCQ often are treated with a combination of HCQ and QC. A study comparing 22 CLE patients who responded to HCQ and 27 CLE patients requiring HCQ and QC (HCQ-QC) found that patients in the HCQQC group had significantly increased number of myeloid dendritic cells and higher expression of TNF- $\alpha$ in their skin lesions compared to the HCQ group. As such, increased myeloid dendritic cell population with higher TNF- $\alpha$ 


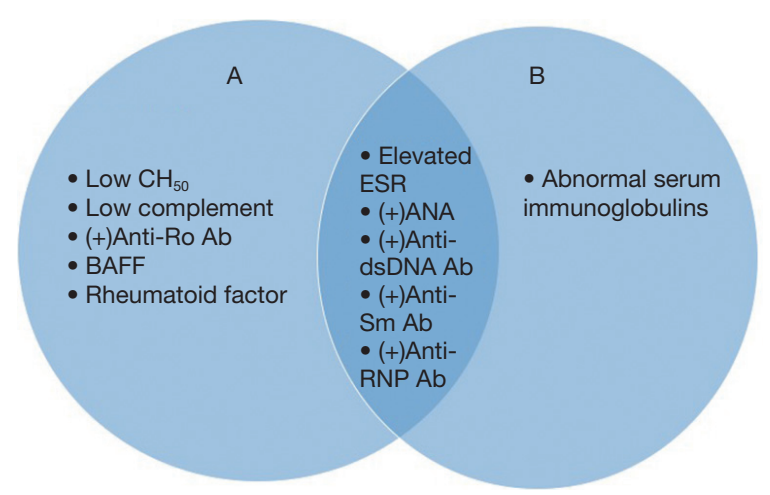

Figure 1 Biomarkers associated with systemic involvement in patients with CLE. Laboratory tests associated with SLE development from studies comparing SLE patients with CLE and CLE-only patients (A), and those comparing CLE patients who develop SLE and DLE-only patients (B). Signs commonly identified from both types of studies are listed in the Venn diagram overlap. Ab, antibody; ANA, antinuclear antibody; BAFF, B-cell activating factor; $\mathrm{CH} 50$, total hemolytic complement; dsDNA, double-stranded DNA; ESR, erythrocyte sedimentation rate; RNP, ribonucleotide protein; CLE, cutaneous lupus erythematosus; SLE, systemic lupus erythematosus. Adapted from Chong et al. 2011 (46).

expression may be predictive of poor treatment response to HCQ in CLE patients (23).

\section{Biomarkers associated with SLE and the progression of CLE to SLE}

Anti-dsDNA antibody, anti-SS-A antibody, ANA, low complement, and BAFF are associated with SCLE and CCLE and a concomitant diagnosis of SLE. Multiple studies have examined CCLE or SCLE patients with and without SLE and identified key serum differences between them. In a retrospective study of 62 DLE patients, six had concomitant diagnoses of DLE and SLE. A positive ANA and dsDNA antibody were found to be associated with DLE and SLE diagnosis as compared to DLE alone (17). Retrospective studies investigating CCLE patients have found that ANA, anti-dsDNA antibody, anti-SS-A antibody, anti-RNP antibody, anti-Sm antibody, rheumatoid factor, elevated erythrocyte sedimentation rate (ESR), and low complement are associated with a concomitant SLE diagnosis $(18,45)$. A retrospective study involving 73 DLE patients found that 10 fulfilled SLE diagnosis, with 8 at baseline and 2 subsequently after 4 and 6 months of initial presentation. A positive ANA was found to correlate with diagnosis of SLE. Additionally, this study noted that in patients with both DLE and SLE, SLE diagnostic criteria was primarily satisfied through mucocutaneous and serological criteria. Serious organ involvement was less frequent and only seen in 20\% of patients with DLE and SLE (21). In another retrospective study, 80 CCLE patients (Group 1) were compared to 15 CCLE patients with at least one systemic lupus manifestation (Group 2) and 13 SLE patients with biopsy-proven nephritis (Group 3). This study identified low CH50 complement to be associated with SLE, as $4 \%$ of CCLE only patients had low $\mathrm{CH} 50$ versus $47 \%$ of patients in group 2 and 3 (19). In a 10-year retrospective study of patients with CLE in Korea, 44 patients with CCLE only and 91 patients with both CCLE and SLE were evaluated. Among this group, patients who had SLE diagnosis were more likely to have positive ANA, anti-dsDNA, anti-Sm, and anti-RNP antibodies. Additionally, they more frequently had an elevated ESR and reduced levels of $\mathrm{C} 3, \mathrm{C} 4$, and $\mathrm{CH} 50$. This study suggests that CCLE patients with presence of these biomarkers have a worse prognosis than those without (24). In DLE, serum BAFF mRNA and protein levels have been found to be higher in DLE patients with concomitant SLE compared to DLE patients without SLE and healthy controls (13). Finally, in a retrospective cohort study involving 112 Caucasian SCLE patients, 46 (41\%) had SLE at the time of SCLE diagnosis. This study found that patients with SCLE and concomitant SLE had elevated ESR, positive ANA, anti-dsDNA antibody, anti-SS-A antibody, anti-RNP antibody, anti-Sm antibody, rheumatoid factor, and low $\mathrm{C} 3$ and CH50 (18).

ANA, anti-dsDNA antibody, anti-Sm antibody, anti-U1 RNP antibody, and elevated ESR may reflect CLE patients who are predisposed to developing SLE. While there are fewer studies comparing CLE patients who did and did not progress to SLE, they have identified similar sera biomarkers, such as autoantibodies against nuclear antigens, and elevated ESR, as studies comparing CLE patients without and with SLE (Figure 1). Positive ANA and antidsDNA antibody have been demonstrated to be associated with DLE progression to SLE in a retrospective study of 130 DLE patients (20). Another retrospective study of a cohort of DLE patients determined that patients that developed SLE persistently had elevated ESR, positive ANA, and abnormal serum immunoglobulins (25). A prospective, longitudinal study of 77 CLE patients found that $13.5 \%$ of patients went on to meet criteria for SLE 
primarily by meeting mucocutaneous ACR criteria. At baseline, patients that developed SLE (SLE $\mathrm{S}_{\mathrm{C}}$ group) were more likely to have a positive ANA and a greater number of ACR criteria compared to patients that remained CLE (CLE group) (47). We recently conducted a retrospective cohort study aimed to identify risk factors that predispose CLE patients to develop SLE. 57 patients remained CLE (CLE-only) while 12 CLE patients progressed to SLE (CLE to SLE). CLE to SLE patients had more likely to have immunologic disorder than CLE-only patients; however, individual autoantibodies and laboratory abnormalities were not significantly higher in these patients (27). In a retrospective study of 40 children with DLE, six had SLE concurrently with their DLE diagnosis while nine progressed from DLE to SLE. Twenty-five patients remained DLE only. Patients with SLE, either concurrently or after progression to SLE, were more likely to present with certain immunologic findings including positive antiSm antibody and anti-U1 RNP antibody (48).

\section{Conclusions}

In summary, the clinical heterogeneity of CLE makes the identification and development of specific biomarkers for CLE challenging. Nonetheless, studies have identified several promising biomarkers such as type I interferonrelated proteins, and anti-SSA-autoantibody that may be informative for the diagnosis of CLE and its subtypes. Type I interferon-inducible proteins can potentially be measured in the sera and skin of CLE patients to assess disease activity. Lastly, autoantibodies against various nuclear antigens, elevated ESR and low complement in CLE patients may reflect poorer prognosis and an increased risk of developing systemic disease. Further larger observational and mechanistic studies are needed to confirm roles of these various candidate biomarkers and their potential role in CLE pathogenesis and heterogeneity.

\section{Acknowledgments}

The authors would like to acknowledge Rose Cannon for her administrative help.

Funding: None.

\section{Footnote}

Provenance and Peer Review: This article was commissioned by the Guest Editors (Drs. Richard D. Sontheimer, M.
Kari Connolly, David F. Fiorentino, and Victoria P. Werth) for the series "Rheumatologic Skin Disease" published in Annals of Translational Medicine. The article has undergone external peer review.

Conflicts of Interest: All authors have completed the ICMJE uniform disclosure form (available at http://dx.doi. org/10.21037/atm-20-5232). The series "Rheumatologic Skin Disease" was commissioned by the editorial office without any funding or sponsorship. BFC reports grants from Daavlin Corporation, other from Pfizer Corporation, other from Biogen Corporation, personal fees from Bristol Meyers Squibb, personal fees from Viela Bio, personal fees from Beacon Bioscience, other from Amgen Incorporated, personal fees from Principia Biopharma, during the conduct of the study. The authors have no other conflicts of interest to declare.

Ethical Statement: The authors are accountable for all aspects of the work in ensuring that questions related to the accuracy or integrity of any part of the work are appropriately investigated and resolved.

Open Access Statement: This is an Open Access article distributed in accordance with the Creative Commons Attribution-NonCommercial-NoDerivs 4.0 International License (CC BY-NC-ND 4.0), which permits the noncommercial replication and distribution of the article with the strict proviso that no changes or edits are made and the original work is properly cited (including links to both the formal publication through the relevant DOI and the license). See: https://creativecommons.org/licenses/by-nc-nd/4.0/.

\section{References}

1. Reeves W, Narain S, Satoh M. Autoantibodies in systemic lupus erythematosus. In: Koopman WJ, Moreland LW. editors. Arthritis and Allied Conditions A Textbook of Rheumatology. Philadelphia: Lippincott Williams and Wilkins, 2005.

2. Kavanaugh A, Tomar R, Reveille J, et al. Guidelines for clinical use of the antinuclear antibody test and tests for specific autoantibodies to nuclear antigens. Arch Pathol Lab Med 2000;124:71-81.

3. Manzi S, Navratil JS, Ruffing MJ, et al. Measurement of erythrocyte $\mathrm{C} 4 \mathrm{~d}$ and complement receptor 1 in systemic lupus erythematosus. Arthritis Rheumatism 2004;50:3596-604. 
4. Panda AK, Parida JR, Tripathy R, et al. Mannose binding lectin: a biomarker of systemic lupus erythematosus disease activity. Arthritis Res Ther 2012;14:R218.

5. Chun HY, Chung JW, Kim HA, et al. Cytokine IL-6 and IL-10 as biomarkers in systemic lupus erythematosus. J Clin Immunol 2007;27:461-6.

6. Bauer JW, Petri M, Batliwalla FM, et al. Interferonregulated chemokines as biomarkers of systemic lupus erythematosus disease activity: a validation study. Arthritis Rheum 2009;60:3098-107.

7. Nabatian AS, Bashir MM, Wysocka M, et al. Tumor necrosis factor $\alpha$ release in peripheral blood mononuclear cells of cutaneous lupus and dermatomyositis patients. Arthritis Res Ther 2012;14:R1.

8. Sarkar MK, Hile GA, Tsoi LC, et al. Photosensitivity and type I IFN responses in cutaneous lupus are driven by epidermal-derived interferon kappa. Ann Rheum Dis 2018;77:1653-64.

9. Wenzel J, Wörenkämper E, Freutel S, et al. Enhanced type I interferon signalling promotes Th1-biased inflammation in cutaneous lupus erythematosus. J Pathol 2005;205:435-42.

10. Braunstein I, Klein R, Okawa J, et al. The interferonregulated gene signature is elevated in subacute cutaneous lupus erythematosus and discoid lupus erythematosus and correlates with the cutaneous lupus area and severity index score. Br J Dermatol 2012;166:971-5.

11. Naschberger E, Wenzel J, Kretz CC, et al. Increased expression of guanylate binding protein-1 in lesional skin of patients with cutaneous lupus erythematosus. Exp Dermatol 2011;20:102-6.

12. Furie R, Werth VP, Merola JF, et al. Monoclonal antibody targeting BDCA2 ameliorates skin lesions in systemic lupus erythematosus. J Clin Invest 2019;129:1359-71.

13. Chong BF, Tseng LC, Kim A, et al. Differential expression of BAFF and its receptors in discoid lupus erythematosus patients. J Dermatol Sci 2014;73:216-24.

14. Walling HW, Sontheimer RD. Cutaneous lupus erythematosus. Am J Clin Dermatol 2009;10:365-81.

15. Pers JO, Daridon C, Devauchelle V, et al. BAFF overexpression is associated with autoantibody production in autoimmune diseases. Ann N Y Acad Sci 2005;1050:34-9.

16. Kim A, O'Brien J, Tseng LC, et al. Autoantibodies and disease activity in patients with discoid lupus erythematosus. JAMA Dermatol 2014;150:651-4.

17. Callen JP. Chronic cutaneous lupus erythematosus: clinical, laboratory, therapeutic, and prognostic examination of 62 patients. Arch Dermatol 1982;118:412-6.

18. Vera-Recabarren M, García-Carrasco M, Ramos-Casals $M$, et al. Comparative analysis of subacute cutaneous lupus erythematosus and chronic cutaneous lupus erythematosus: clinical and immunological study of 270 patients. Br J Dermatol 2010;162:91-101.

19. Prystowsky SD, Gilliam JN. Discoid lupus erythematosus as part of a larger disease spectrum: Correlation of clinical features with laboratory findings in lupus erythematosus. Arch Dermatol 1975;111:1448-52.

20. Insawang M, Kulthanan K, Chularojanamontri L, et al. Discoid lupus erythematosus: description of 130 cases and review of their natural history and clinical course. J Clin Immunol Immunopathol Res 2010;2:1-8.

21. Ng PP, Tan SH, Koh ET, et al. Epidemiology of cutaneous lupus erythematosus in a tertiary referral centre in Singapore. Australas J Dermatol 2000;41:229-33.

22. Tsoi LC, Gharaee-Kermani M, Berthier CC, et al. IL18containing 5-gene signature distinguishes histologically identical dermatomyositis and lupus erythematosus skin lesions. JCI Insight 2020;5:e139558.

23. Zeidi M, Kim HJ, Werth VP. Increased myeloid dendritic cells and TNF- $\alpha$ expression predicts poor response to hydroxychloroquine in cutaneous lupus erythematosus. J Invest Dermatol 2019;139:324-32.

24. Oh EH, Kim EJ, Ro YS, et al. Ten-year retrospective clinicohistological study of cutaneous lupus erythematosus in Korea. J Dermatol 2018;45:436-43.

25. Healy E, Kieran E, Rogers S. Cutaneous lupus erythematosus - a study of clinical and laboratory prognostic factors in 65 patients. Ir J Med Sci 1995;164:113.

26. Sontheimer RD, Maddison PJ, Reichlin M, et al. Serologic and HLA associations in subacute cutaneous lupus erythematosus, a clinical subset of lupus erythematosus. Ann Intern Med 1982;97:664-71.

27. Walocko F, Black S, Anderson S, Li X, et al. Worsening skin damage in patients with cutaneous lupus erythematosus may predict development of systemic lupus erythematosus. J Am Acad Dermatol 2021;84:538-40.

28. Fäh J, Pavlovic J, Burg G. Expression of MxA protein in inflammatory dermatoses. J Histochem Cytochem $1995 ; 43: 47-52$.

29. Haller O, Kochs G. Interferon-induced mx proteins: dynamin-like GTPases with antiviral activity. Traffic 2002;3:710-7.

30. Iijima $W$, Ohtani $H$, Nakayama T, et al. Infiltrating CD8+ $\mathrm{T}$ cells in oral lichen planus predominantly express CCR5 
and CXCR3 and carry respective chemokine ligands RANTES/CCL5 and IP-10/CXCL10 in their cytolytic granules: a potential self-recruiting mechanism. Am J Pathol 2003;163:261-8.

31. Padovan E, Spagnoli GC, Ferrantini M, et al. IFN- $\alpha 2 a$ induces IP-10/CXCL10 and MIG/CXCL9 production in monocyte-derived dendritic cells and enhances their capacity to attract and stimulate CD8+ effector T cells. J Leukoc Biol 2002;71:669-76.

32. Naschberger E, Werner T, Vicente AB, et al. Nuclear factor-kappaB motif and interferon-alpha-stimulated response element co-operate in the activation of guanylatebinding protein-1 expression by inflammatory cytokines in endothelial cells. Biochem J 2004;379:409-20.

33. Lubeseder-Martellato C, Guenzi E, Jörg A, et al. Guanylate-binding protein-1 expression is selectively induced by inflammatory cytokines and is an activation marker of endothelial cells during inflammatory diseases. Am J Pathol 2002;161:1749-59.

34. Cheng YS, Colonno RJ, Yin FH. Interferon induction of fibroblast proteins with guanylate binding activity. J Biol Chem 1983;258:7746-50.

35. Kretz CC, Norpo M, Abeler-Dörner L, et al. Anti-annexin 1 antibodies: A new diagnostic marker in the serum of patients with discoid lupus erythematosus. Exp Dermatol 2010;19:919-21.

36. Chong BF, Tseng LC, Lee T, et al. IgG and IgM autoantibody differences in discoid and systemic lupus patients. J Invest Dermatol 2012;132:2770-9.

37. Davidson A. Targeting BAFF in autoimmunity. Curr Opin Immunol 2010;22:732-9.

38. Chu VT, Enghard P, Schürer S, et al. Systemic activation of the immune system induces aberrant BAFF and APRIL expression in $B$ cells in patients with systemic lupus erythematosus. Arthritis Rheum 2009;60:2083-93.

39. Cheema GS, Roschke V, Hilbert DM, et al. Elevated serum B lymphocyte stimulator levels in patients with

Cite this article as: Zhu JL, Black SM, Chong BF. Role of biomarkers in the diagnosis and prognosis of patients with cutaneous lupus erythematosus. Ann Transl Med 2021;9(5):429. doi: 10.21037/atm-20-5232 systemic immune-based rheumatic diseases. Arthritis Rheum 2001;44:1313-9.

40. Stohl W, Metyas S, Tan SM, et al. B lymphocyte stimulator overexpression in patients with systemic lupus erythematosus: longitudinal observations. Arthritis Rheum 2003;48:3475-86.

41. Yao Y, Richman L, Higgs BW, et al. Neutralization of interferon- $\alpha / \beta$-inducible genes and downstream effect in a phase I trial of an anti-interferon- $\alpha$ monoclonal antibody in systemic lupus erythematosus. Arthritis Rheum 2009;60:1785-96.

42. Lesiak A, Narbutt J, Kobos J, et al. Systematic administration of chloroquine in discoid lupus erythematosus reduces skin lesions via inhibition of angiogenesis. Clin Exp Dermatol 2009;34:570-5.

43. Chang AY, Piette EW, Foering KP, et al. Response to antimalarial agents in cutaneous lupus erythematosus: a prospective analysis. Arch Dermatol 2011;147:1261-7.

44. Wahie S, Daly AK, Cordell HJ, et al. Clinical and pharmacogenetic influences on response to hydroxychloroquine in discoid lupus erythematosus: a retrospective cohort study. J Invest Dermatol 2011;131:1981-6.

45. Vera-Recabarren MA, García-Carrasco M, Ramos-Casals $\mathrm{M}$, et al. Cutaneous lupus erythematosus: clinical and immunological study of 308 patients stratified by gender. Clin Exp Dermatol 2010;35:729-35.

46. Chong BF, Song J, Olsen NJ. Determining risk factors for developing systemic lupus erythematosus in patients with discoid lupus erythematosus. Br J Dermatol 2012;166:29-35.

47. Wieczorek IT, Propert KJ, Okawa J, et al. Systemic symptoms in the progression of cutaneous to systemic lupus erythematosus. JAMA Dermatol 2014;150:291-6.

48. Arkin LM, Ansell L, Rademaker A, et al. The natural history of pediatric-onset discoid lupus erythematosus. J Am Acad Dermatol 2015;72:628-33. PubMed 